JOURNAL of

MAINE MEDICAL CENTER Journal of Maine Medical Center

Volume 2

Issue 2 Volume 2, Issue 2 (July 2020)

Article 13

2020

\title{
A case of Disseminated Nocardia beijingensis in an Immunocompetent Patient, Manifesting as Subcutaneous and CNS Nocardiosis.
}

\author{
Scott Cathcart \\ Maine Medical Center
}

Et al.

Follow this and additional works at: https://knowledgeconnection.mainehealth.org/jmmc

Part of the Bacterial Infections and Mycoses Commons, Infectious Disease Commons, and the Nervous System Diseases Commons

\section{Recommended Citation}

Cathcart, Scott; Stogsdill, Patricia; and Bell, Rebecca (2020) "A case of Disseminated Nocardia beijingensis in an Immunocompetent Patient, Manifesting as Subcutaneous and CNS Nocardiosis.," Journal of Maine Medical Center. Vol. 2 : Iss. 2 , Article 13.

Available at: https://knowledgeconnection.mainehealth.org/jmmc/vol2/iss2/13 https://doi.org/10.46804/ 2641-2225.1057

The views and thoughts expressed in this manuscript belong solely to the author[s] and do not reflect the opinions of the Journal of Maine Medical Center or MaineHealth.

This Case Report is brought to you for free and open access by Maine Medical Center Department of Medical Education. It has been accepted for inclusion in the Journal of Maine Medical Center by an authorized editor of the MaineHealth Knowledge Connection. For more information, please contact Dina McKelvy mckeld1@mmc.org.

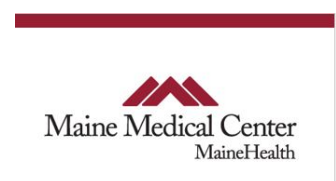


A case of Disseminated Nocardia beijingensis in an Immunocompetent Patient, Manifesting as Subcutaneous and CNS Nocardiosis.

Acknowledgements

None

Authors

Scott Cathcart, Patricia Stogsdill, and Rebecca Bell 


\title{
A Case of Disseminated Nocardia beijingensis Manifesting as Subcutaneous and CNS Nocardiosis in an Immunocompetent Patient
}

\author{
Scott Cathcart, MD, ${ }^{1}$ Patricia Stogsdill, MD, ${ }^{2}$ Rebecca Bell ${ }^{3}$ \\ ${ }^{1}$ Department of Internal Medicine, Maine Medical Center, Portland, ME, ${ }^{2}$ Department of Infectious Diseases, Maine Medical \\ Center, Portland, ME, ${ }^{3}$ Tufts Medical School, Boston, MA
}

\begin{abstract}
Introduction: Nocardiosis is an uncommon infection, typically encountered in immunocompromised patients. Only 5 cases have been reported in the United States. We present a case of disseminated Nocardia beijingensis involving the lung, subcutaneous tissue, and central nervous system of an immunocompetent patient.
\end{abstract}
Clinical Findings: A 60-year-old immunocompetent male with no significant medical history presented to our hospital after a complicated medical course, beginning with vague respiratory complaints. His initial work-up revealed a large mass of unclear etiology in the right upper lung. A month later, he had a syncopal episode and then developed focal neurologic deficits.

\begin{abstract}
Diagnosis, Sputum cultures obtained after the patient's syncopal episode grew gram-positive rods. Results of a Interventions, computed tomography of the chest showed a new $17-\mathrm{mm}$ subcutaneous mass. The mass was biopsied, and Outcomes: which revealed gram-positive rods with partial acid-fast staining. Week later, results of magnetic resonance imaging of his brain showed multiple enhancing lesions with vasogenic edema scattered throughout. A tissue culture from his chest mass was sent to the Mayo Clinic and ultimately grew Nocardia beijingensis. The patient was started on the appropriate antibiotic therapy and has made significant clinical improvement.
\end{abstract}

Conclusions: Though Nocardia is often associated with an immunocompromised state, it can also occur in patients with pulmonary findings and evidence of disseminated disease, particularly central nervous system manifestations, regardless of immune status. A high index of suspicion is needed, as definitive diagnosis often takes weeks due to the slow-growing nature of Nocardia species.

Keywords: $\quad$ Nocardia beijingensis, disseminated, neurologic, CNS

\section{CASE PRESENTATION}

The patient was a pleasant, albeit anxious, gentleman with a greater than 20-year history of right-side neck pain following a remote trauma, as well as post-traumatic stress disorder. In March 2019, the patient was seen by his primary care physician after 2 months of progressive cough with associated wheezing, fevers, sweats, and chest/back pain. The results of his chest $\mathrm{x}$-ray were negative, but the results of his follow-up CT

Correspondence: Scott Cathcart, MD

Department of Internal Medicine, Maine Medical Center 22 Bramhall Street, Portland, ME 04102

scott.a.cathcart@gmail.com (computed tomography) revealed a large mass in his right apical lung (Figure 1).

Three days after the CT, the patient was evaluated in the emergency department (ED), where his health care provider was initially concerned about malignancy vs tuberculosis. The results of a T-spot were negative. He was given amoxacillinclavulanate for post-obstructive pneumonia. Two days later, the results of his follow-up PET (positron emission tomography) scan showed a 6- to 7-cm mass in his right upper lobe that was strongly positive for fluorodeoxyglucose. This result suggested a primary lung malignancy/Pancoast tumor, especially in the context of fevers, night sweats, and weight loss. Interestingly, he showed 


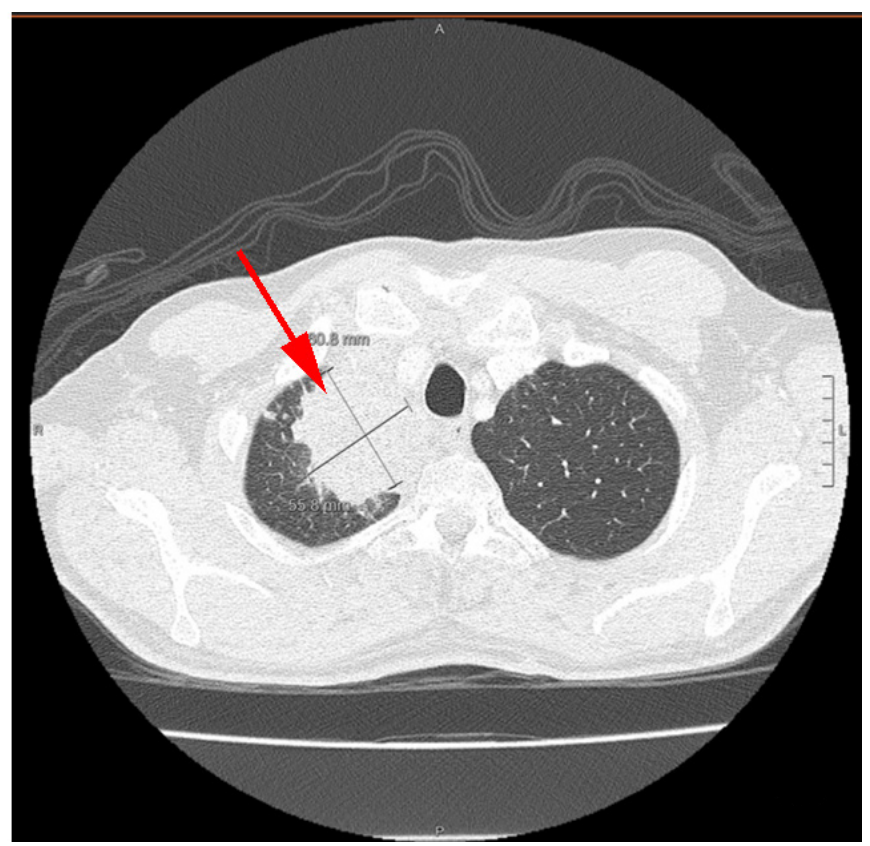

Figure 1. Computed tomography of the chest revealing large mass in right apical lung.

a lack of mediastinal adenopathy. The results of a subsequent CT-guided biopsy revealed chronic inflammatory changes consistent with pneumonia and no evidence of neoplasm. He failed to improve with amoxacillin-clavulanate. He was transitioned to levofloxacin and a prednisone taper due to concern for bronchiolitis obliterans organizing pneumonia, and he continued his 3-week course of antibiotics. This treatment appeared to provide some benefit, but his condition deteriorated when antibiotics were stopped. A bronchoscopy was performed 1 month after his initial presentation to the ED, but the results were negative for Pneumocystis jiroveci, as were the results following fungal silver stain, cytology for malignancy, and acid-fast staining and culture. He was given a 5-day steroid burst after the procedure, again without improvement.

He presented to the ED again after a syncopal episode several days after the bronchoscopy with a leukocytosis of 30,000 ( $90 \%$ polymorphonuclear), fevers to $102^{\circ} \mathrm{F}$, and complaints of intermittent hemoptysis. The patient was given broad-spectrum antibiotics (meropenem and vancomycin) as his presentation was again thought to be due to a bacterial process, and he had responded positively to antibiotics in the past. Sputum cultures eventually grew gram-positive rods. The results of a CT of the chest during this stay revealed a stable infiltrate in the right upper lung with new tree-in-bud opacities and mild ground-glass opacities, consistent with multifocal pneumonia. The results also noted a new 17-mm subcutaneous mass on his chest at the level of the sternum. Results from a biopsy of this lesion revealed gram-positive rods with partial acid-fast staining, concerning for Actinomyces or Nocardia, similar to those identified in the sputum cultures obtained on his admission. The culture was sent to the Mayo Clinic for speciation and susceptibility testing. Given the patient's immunocompetent state (he was HIV-negative and lacked other comorbid conditions), Actinomyces was favored over Nocardia, and he was given IV penicillin in addition to meropenem. This treatment was further tailored to oral trimethoprim-sulfamethoxazole (800/160 mg, 2 times daily) and penicillin $V(500 \mathrm{mg}, 4$ times daily) before discharging him on hospital day 10 . He was discharged with plans for indefinite antibiotics and follow-up with his pulmonologist. During this admission, an extensive work-up for possible fungal etiologies was performed. The results were negative for Cryptococcus, coccidioidomycosis, and histoplasmosis. A CT of his head was also conducted to further evaluate the patient's syncope. The result of the CT showed 2 discrete focal hypodensities within the right and left parietal graywhite junctions with surrounding edema, which were concerning for metastatic disease vs focal areas of hemorrhage. This finding was not worked up further.

The patient initially did well on this regimen. At a follow-up appointment with his pulmonologist a week after discharge, he reported feeling much better, his fevers and hemoptysis resolved, and he noticed a decrease in his night sweats. However, his cough persisted. He subsequently developed issues with balance and double-vision, particularly with an extreme upward gaze, which prompted his return to the ED a week later. The results of an MRI of his brain revealed "multiple enhancing lesions with vasogenic edema scattered throughout," which was consistent with an infectious process in his central nervous system (CNS), with disseminated Nocardia the most likely organism (Figure 2).

He was given IV meropenem, IV trimethoprimsulfamethoxazole, and steroids, and then transferred to our facility for a higher level of care. A transesophageal echo was obtained to rule out endocarditis. An MRI of his spine was performed to look for osteomyelitis. Results of imaging of his cervical spine raised the possibility of osteomyelitis with abnormal prevertebral edema at the level of C5/C6, which was not seen in the results of an 


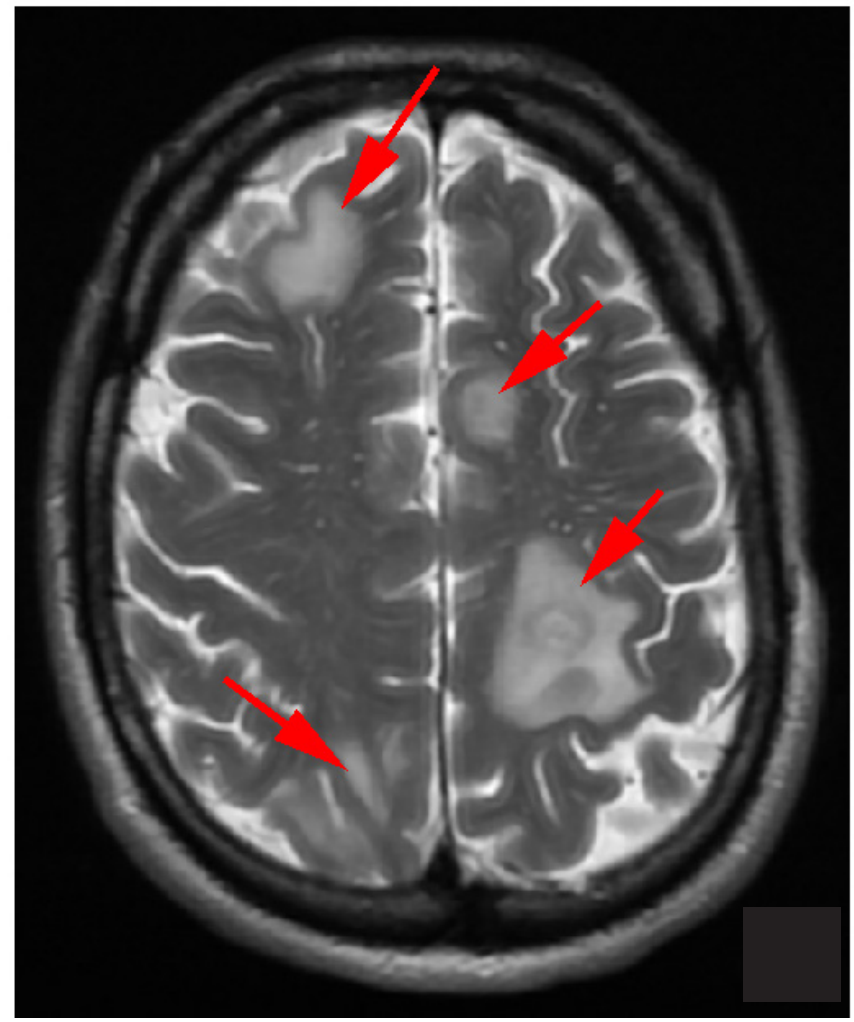

Figure 2. T2-weighted magnetic resonance imaging of the brain with multiple enhancing lesions and vasogenic edema

MRI obtained several months earlier. A peripherally inserted central catheter line was placed, and the patient was discharged on 6 weeks of IV antibiotics (trimethoprim-sulfamethoxazole and meropenem) and close follow-up with infectious diseases. Culture data returned from the Mayo Clinic, which revealed Nocardia beijingensis. After completing 5 weeks of IV antibiotics, he was transitioned to per os trimethoprim-sulfamethoxazole with a plan to continue therapy for an additional 12 months.

Two months after his diagnosis (almost 4 months after his initial ED presentation), the patient ceased to experience night sweats or cough, and he began to regain strength and daily functioning. However, his posterior neck pain and radicular symptoms down his right side persisted. The results of a followup chest CT at this time showed that the mass-like consolidation in the right upper lobe had nearly resolved. The results of an MRI of his brain 1 month later revealed a marked interval decrease in the size and number of enhancing parenchymal lesions when compared to results from 2 months earlier. The adjacent vasogenic edema also significantly decreased. The results of an MRI of the spine at the same time was consistent with ongoing Nocardia osteomyelitis, with decreased prevertebral edema and no evidence of discitis. The patient continued to have double-vision at the periphery as a result of these resolving brain lesions. Five months later, the results of another MRI of the brain showed a further decrease in size and signal intensity of the focal areas of signal hyperintensity, as well as resolution of signal enhancement, compared to prior imaging.

\section{DISCUSSION}

The genus Nocardia is a ubiquitous group of grampositive bacilli found in the environment, primarily in soil, but also in decomposing organic matter and both fresh water and saltwater. These bacteria are thought to play a role in the soil ecosystem and can cause significant disease in susceptible hosts. ${ }^{1,2}$ More than 50 species have been identified. Of these, the most common are the Nocardia asteroides complex, which have been reorganized into several different species based on their differing drug susceptibilities. ${ }^{1}$ Nocardia species are typically opportunistic organisms, but up to onethird of patients are immunocompetent. Pulmonary nocardiosis is the most common presentation, as inhalation is the primary route of inoculation, and is often difficult to differentiate from fungal and mycobacterial infections. Extrapulmonary manifestations due to disseminated disease are common. The most common sites for dissemination include the CNS, skin and subcutaneous tissues, eyes, kidneys, joints, bones, and the heart. The CNS is affected in approximately one-third of all cases. Primary cutaneous and soft-tissue nocardial lesions can occur following traumatic skin injuries contaminated with soil, typically in immunocompetent hosts. ${ }^{1,3}$

In 2001, Nocardia beijingensis was first identified after being isolated from a mud sample taken from a sewage ditch in Beijing. ${ }^{2}$ In 2004, the first human infections were reported in Thailand and Japan. ${ }^{4}$ In 2014, the first case of Nocardia beijingensis was reported in the United States. The case involved an immunocompetent patient at the Mayo Clinic Florida. ${ }^{5}$ Since 2014, only 5 additional cases of Nocardia beijingensis have been reported in the Western hemisphere. Of these, 3 occurred in immunocompetent hosts, and all 3 were in Florida. ${ }^{6-10}$ The most recent report by Chauhan, in 2019, was the only case in the United States that involved the CNS. ${ }^{9}$ Ours is the second reported case of CNS nocardiosis in the United States, and the first with multiple simultaneous manifestations of disseminated nocardiosis. Our immunocompetent 
patient developed primary pulmonary disease, which spread to the subcutaneous tissue overlying his sternum and to his brain. The diagnosis was ultimately made based on a tissue biopsy of his sternal lesion, and the CNS lesions were assumed to be of the same etiology.

Pulmonary nocardiosis typically presents as subacute pneumonia, with symptoms developing over days or weeks. Less common is an acute presentation with symptoms such as dyspnea, pleuritic pain, and hemoptysis. ${ }^{11}$ CNS nocardiosis is also variable in presentation, most often manifesting as focal neurologic deficits, headache, fever, and seizures. CNS nocardiosis appears as ring-enhancing lesions on brain imaging and is often initially misdiagnosed as tumor or abscess. ${ }^{12}$

Clinical diagnosis is difficult, given the varied and nonspecific presentations. The current diagnostic gold standard is a positive microbiological culture from a site of active infection. The results of most cultures are positive within 2 to 7 days, but they can take up to 2 to 3 weeks due to the bacteria's slow growth. ${ }^{13}$ Testing by polymerase chain reaction is currently being evaluated but not generally available in practice. ${ }^{14}$ Both Nocardia and Actinomyces fail to stain with classic, non-modified acid-fast stains. However, Nocardia partially stains with acid-fast staining when modified techniques are used. This approach is useful for differentiating Nocardia from Actinomyces, another gram-positive, thin, branching, filamentous bacilli. Actinomyces may present similarly to Nocardia, but the results of modified acid-fast staining are classically negative for Actinomyces. ${ }^{15}$ This fact suggests that the initial staining results from the chest lesion should have been interpreted as more concerning for Nocardia than Actinomyces. However, there have been rare reports of Actinomyces staining positive with modified acid-fast staining. ${ }^{16}$ Another useful test to differentiate these 2 organisms is anaerobic culture. Actinomyces species will grow under anaerobic conditions, but Nocardia are obligate aerobes. ${ }^{15}$ In our case, an anaerobic culture of the chest lesion may have helped providers arrive at the correct diagnosis sooner.

First-line treatment for nocardiosis is sulfonamide therapy, with trimethoprim-sulfamethoxazole being the most commonly used agent. Several alternative choices are effective against most https://knowledgeconnection.mainehealth.org/jmmc/vol2/iss2/13 DOI: $10.46804 / 2641-2225.1057$
Nocardia species, including amikacin, imipenem, meropenem, ceftriaxone, cefotaxime, minocycline, moxifloxacin, levofloxacin, linezolid, tigecycline, and amoxicillin-clavulanic acid. In most cases, combination therapy should be used to enhance activity and decrease the possibility of developing antibiotic resistance. In treating CNS disease, antibiotics with favorable CNS penetration should be selected. Sulfonamides, alone or combined with trimethoprim, are most effective and typically used first-line for at least 1 year. Combining minocycline, imipenem, or aminoglycoside with a third-generation cephalosporin may also be used as alternative therapies. ${ }^{17}$

This case illustrates that a diagnostic dilemma can arise when a rare condition presents in an atypical population. Our patient presented with an unusual infection and no clear exposure history. Furthermore, the organism is more commonly seen in immunocompromised hosts and is less likely to present in immunocompetent patients. This detail clearly affected the interpretation of the culture data obtained from the patient's chest mass, delaying his appropriate treatment. While Nocardia is less common, it is important to remember that more than $30 \%$ of patients infected with Nocardia are immunocompetent. Finally, multiple tests can help differentiate Actinomyces from Nocardia when the diagnosis is unclear and should be used when possible.

\section{Conflict of Interest: None}

\section{REFERENCES}

1. Wilson JW. Nocardiosis: updates and clinical overview. Mayo Clin Proc. 2012;87(4):403-407. doi:10.1016/j.mayocp.2011.11.01.

2. Wang L, Zhang Y, Lu Z, et al. Nocardia beijingensis sp. nov., a novel isolate from soil. Int $J$ Syst Evol Microbiol. 2001;51(Pt 5):1783-1788. doi:10.1099/00207713-51-5-1783.

3. Lerner PI. Nocardiosis. Clin Infect Dis. 1996;22(6):891-903; quiz 904-905. doi:10.1093/clinids/22.6.891.

4. Kageyama A, Poonwan N, Yazawa K, Mikami Y, Nishimura K. Nocardia beijingensis, is a pathogenic bacterium to humans: the first infectious cases in Thailand and Japan. Mycopathologia. 2004;157(2):155-161. doi:10.1023/ b:myco.0000020588.60081.37.

5. Crozier JA, Andhavarapu S, Brumble LM, Sher T. First report of Nocardia beijingensis infection in an immunocompetent host in the United States. J Clin Microbiol. 2014;52(7):2730-2732. doi:10.1128/JCM.00588-14.

6. Solano-Varela DM, Barrios-Vidales EM, Plaza DF, et al. Immunocompetent patient with a brain abscess caused by Nocardia beijingensis in Latin America: A case report. Medicine (Baltimore). 2019;98(11):e14879. doi:10.1097/ MD.0000000000014879. 


\section{Cathcart et al.: Disseminated Nocardia beijingensis}

7. Keenan JG, Mohapatra S. Nocardia beijingensis brain abscesses in an HIV-infected individual. IDCases. 2017;9:65-69. doi:10.1016/j.idcr.2017.03.006.

8. Gonzalez A, Jennings E, Vaziri S, Yachnis AT, Kubal A. Second report of a Nocardia beijingensis infection in the United States: nodular scleritis with in vitro imipenem resistance. Digit $J$ Ophthalmol. 2016;22(3):62-66. doi:10.5693/djo.02.2016.01.003.

9. Chauhan L, Vassa N, Henderson E, Mubarik A, Siddiq D, Eddib A. 1416. Nocardia beijingensis: A Rare and Unusual Cause of Intracranial Abscess. Open Forum Infect Dis. 2019;6(Suppl 2):S515-S516. doi:10.1093/ofid/ofz360.1280.

10. Aragaki-Nakahodo A, Benzaquen S, Kirschner M. Coinfection by Nocardia beijingensis and Nocardia arthritidis in an immunocompromised patient diagnosed by endobronchial ultrasound guided transbronchial needle aspiration (EBUSTBNA). Respir Med Case Rep. 2014;12:22-23. doi:10.1016/j. rmcr.2013.11.001.

11. Singh AK, Shukla A, Bajwa R, Agrawal R, Srivastwa N. Pulmonary Nocardiosis: Unusual Presentation in Intensive Care Unit. Indian J Crit Care Med. 2018;22(2):125-127. doi:10.4103/ ijccm.IJCCM_472_17.

12. Anagnostou T, Arvanitis M, Kourkoumpetis TK, Desalermos A, Carneiro HA, Mylonakis E. Nocardiosis of the central nervous system: experience from a general hospital and review of 84 cases from the literature. Medicine (Baltimore). 2014;93(1):1932. doi:10.1097/MD.0000000000000012.

13. Saubolle MA, Sussland D. Nocardiosis: review of clinical and laboratory experience. J Clin Microbiol. 2003;41(10):4497-4501. doi:10.1128/jcm.41.10.4497-4501.2003.

14. Rouzaud C, Rodriguez-Nava V, Catherinot E, et al. Clinical Assessment of a Nocardia PCR-Based Assay for Diagnosis of Nocardiosis. J Clin Microbiol. 2018;56(6):e00002-e00018. doi:10.1128/JCM.00002-18.

15. McHugh KE, Sturgis CD, Procop GW, Rhoads DD. The cytopathology of Actinomyces, Nocardia, and their mimickers. Diagn Cytopathol. 2017;45(12):1105-1115. doi:10.1002/ dc. 23816.

16. Lowe RN, Azimi PH, McQuitty J. Acid-fast actinomyces in a child with pulmonary actinomycosis. J Clin Microbiol. 1980;12(1):124-126. Accessed April 11, 2020. https://jcm.asm. org/content/12/1/124.long.

17. Mamelak AN, Obana WG, Flaherty JF, Rosenblum ML. Nocardial brain abscess: treatment strategies and factors influencing outcome. Neurosurgery. 1994;35(4):622-631. doi:10.1227/00006123-199410000-00007. 